\title{
Flame Retardant Waterborne Polyurethanes: Synthesis, Characterization, and Evaluation of Different Properties
}

\author{
Parth Kapatel 1,*(i), Rasmika Patel 1,*(D) \\ 1 Department of Materials Science, Sardar Patel University, Vallabh Vidyanagar, 388120, Gujarat, India; \\ parth91kapatel@gmail.com (P.K.); rasmi29@yahoo.com (R.P.) \\ * Correspondence: parth91kapatel@gmail.com; rasmi29@yahoo.com;
}

Scopus Author ID 57190935934

Received: 3.07.2021; Revised: 30.07.2021; Accepted: 2.08.2021; Published: 8.08.2021

\begin{abstract}
A series of flame retardant waterborne polyurethanes (WBPUs) with varying NCO:OH mole ratio, i.e., isocyanate:hydroxyl groups, was synthesized using phosphorus-based polyester polyol and isophorone diisocyanate (IPDI). The phosphorus moiety was kept in the polymeric chain, which improves flame retardancy of the WBPU films. The prepolymer mixing process was adopted for the synthesis route of WBPU systems. The presence of structural-functional groups was confirmed by Fourier transform infrared spectroscopy and nuclear magnetic resonance spectroscopy. The thermal stability and percentage char yield were observed by TGA analysis. As a consequence of the existence of phosphorous moiety, these WBPUs also exhibit flame retardancy, which was examined by limiting oxygen index (LOI) and UL-94 test. All the samples show an increment in thermal stability with an increase in NCO:OH ratio. Maximum flame retardancy was obtained in FWP5 and FWP6 systems with LOI value of $32 \%$.
\end{abstract}

Keywords: waterborne; flame retardancy; polyurethanes; thermal stability; limiting oxygen index.

(c) 2021 by the authors. This article is an open-access article distributed under the terms and conditions of the Creative Commons Attribution (CC BY) license (https://creativecommons.org/licenses/by/4.0/).

\section{Introduction}

The waterborne polyurethanes (WBPUs) have become a matter of apex research for the steep reduction in the cost of the WBPUs and to manage the volatiles during and after the casting. WBPUs offers a wide spectrum of advantages like environmental friendliness, appreciable molecular weight, rigidity, tailored properties of the cast, controlled viscosity, and user-friendly applicability [1-5]. Controllable chemical formulation and excellent properties of WBPUs have led to a many-fold increment in the application sectors for the usability of WBPUs. There are various ways of combining polyols and diisocyanates in order to produce a tailored waterborne polyurethane product [6-10]. Most recently, there is major attention given to impart flame retardancy in polyurethanes, and it has become a principal aim [11]. Hence, the most beneficial way is to add phosphorous moiety to enhance flame retardancy. There are two ways to deal with flame retardancy in polymers, known as the 'additive' type and the "reactive" type [12-15]. From the various literature, conclusions have been made that flame retardancy of the waterborne polyurethanes improved through incorporating additives like phosphorus [16], organo-modified montmorillonite (O-MMT) [17], carbon nanotubes [18], silicone [19], phosphorus, and nitrogen [20], etc. into the WBPUDs. By means of this, thermal stability and flame retardancy can be easily improved. Presently, various issues like poor compatibility, leaching, and a reduction in mechanical properties come into observation. 
Therefore, the utilization of reactive type flame retardants through copolymerization with a flame retardant moiety in the main polymeric chain can resolve these problems, i.e., compatibility, thermal stability, flame retardancy, and poor mechanical property [21]. Patents around the world reveal that halogen-containing flame retardants exhaust corrosive smoke and toxic gases upon combustion. Henceforth, there is exponential demand for flame retardant systems, which ought to be halogen-free for convenient use in WBPU [22]. To attain highperformance polymer materials, the degradation process allows the formulation of the optimal parameters and conditions to design WBPUs. Thermal degradation studies of WBPUs are concluded in many decomposition steps involving the formation of various gaseous products. Therefore, to evaluate the thermal degradation properties, thermogravimetric analysis (TGA) is a suitable method for different types of waterborne polyurethane systems [23-28].

For preparing waterborne polyurethane dispersions, various processes like the prepolymer mixing process, acetone process, melt dispersion process, and ketamine-ketazine processes are existing. In the prepolymer mixing process [29], prepolymer, which is hydrophilically modified, is mixed with isocyanates followed by water. Chain extension then proceeded with amines in the dispersion medium. In the acetone process [30], acetone is to be chosen as a solvent medium in which the hydrophilic isocyanate prepolymer is dissolved, followed by chain extension with diamines and water. As the water starts to add into the prepolymer system, solvent starts to remove from the charge, resulting in pure waterborne dispersion. Melt dispersion process [31], ketamine-ketazine processes, and other processes [32] are also existing, but these two processes, i.e., prepolymer mixing process and acetone process, are extensively used in the production of waterborne dispersions.

In this work, the prepolymer mixing method was adopted [32]. Phosphorous containing polyester polyol was reacted with isophorone diisocyanate, which results in the isocyanateterminated prepolymer. This prepared prepolymer was neutralized with triethylamine. After constant stirring for 30 minutes, a certain amount of water was added, and a chain-extension process was carried out to form the dispersion. In this way, phosphorus-containing WBPUs have been prepared. Various analytical and instrumental methods have been used for characterization. The effect of phosphorus content and varying NCO:OH mole ratio on the thermal stability and degradation of the waterborne polyurethanes have been investigated.

\section{Material and Methods}

\subsection{Materials.}

The reagents resorcinol, phosphorous oxychloride $\left(\mathrm{POCl}_{3}\right), \mathrm{N}, \mathrm{N}$ - dimethyl aniline, adipic acid, xylene, methyl-ethyl ketone(MEK), p-toluene sulphonic acid, polyethylene-glycol (PEG-M.W.400), isophorone diisocyanate(IPDI), dibutyltin dilaurate (DBTDL) were procured from Sigma Aldrich. Triethylamine (TEA), diethylenetriamine (DETA) were obtained from Himedia laboratories, Mumbai. These reagents were used as such without refinement. For the synthesis of dispersions, deionized water was used. Teflon mold was used for casting flame retardant waterborne polyurethane dispersions. 
2.2. Synthesis of polyester-polyol based on phosphorous-containing Tris (m-hydroxyphenyl) monophosphate (THPP).

Tris (m-hydroxyphenyl) monophosphate [33] (1.0 mole), adipic acid (3.0 mole), and polyethylene-glycol (PEG 400) (2.0 mole) were charged in a three-necked round bottom flask equipped with dean \& stark apparatus and condenser. As a catalyst, p-toluene sulphonic acid was used, and xylene was the reaction medium. Initially, the reactants were heated to $120^{\circ} \mathrm{C}$ for 4 hours, and the further reaction was carried out between $195^{\circ} \mathrm{C}$ to $200^{\circ} \mathrm{C}$ temperature. The conversion of polyester-polyol was monitored by determining an acid number with respect to time. When the acid value has reached a constant of $20 \mathrm{mg} \mathrm{KOH} / \mathrm{gm}$ of resin, the reaction was stopped. A pure viscous liquid product was obtained by vacuum distillation. Finally, polyester polyol was taken out into a glass bottle. The reaction scheme for the synthesis of polyesterpolyol based on phosphorous-containing Tris (m-hydroxyphenyl) monophosphate (THPP) is shown in Figure 1.

\subsection{Synthesis of flame retardant waterborne polyurethane dispersion.}

In a four-necked flask, the polyester-polyol (1.0 mole) was taken and dissolved in a methyl ethyl ketone solvent with stirring at room temperature. The flask was equipped with a thermometer, overhead stirrer, reflux condenser and heated up to $75^{\circ} \mathrm{C}$ temperature in a water bath under continuous stirring. At this stage, over a time of 30 minutes in the presence of dibutyltin dilaurate (DBTDL) catalyst (0.05 mole), isophorone diisocyanate (1.5 mole) was added in the reaction flask drop by drop. The percentage NCO content during the reaction was determined using the dibutyl amine back-titration method [34]. Heating proceeded to the point that the \%NCO content came to 2-3\%. Prepolymer was then cooled to room temperature, and a required amount of triethylamine (TEA) was added. To neutralize the carboxylic group, stirring was continued for 30 minutes. A calculated quantity of deionized water was added dropwise under vigorous stirring. After that, diethylenetriamine (DETA) was added for the chain extension process. Final dispersion has a $\mathrm{pH}$ between 7.0 to 8.0. Similarly, other dispersions with the NCO:OH mole ratios of 1.6:1, 1.7:1, 1.8:1, 1.9:1, and 2.0:1 were prepared. The reaction scheme for the synthesis of flame retardant waterborne polyurethane dispersion is illustrated in Figure 4. Experimental arrangement for the synthesis of waterborne polyurethane is shown in Figure 2. Pictographic representations of synthesized flame retardant waterborne polyurethane dispersions (FWBPUDs) and films are shown in Figure 3.

\section{Characterization}

To determine the number average molecular weight, weight average molecular weight, and molecular weight distribution of polymers, the Gel Permeation Chromatographic (GPC) technique was used. GPC instrument, Perkin Elmer USA make with Model Turbo matrix - 40 was used for analysis. FTIR spectra were recorded on ATR- FTIR spectrometer, Bruker, ALPHA model, Germany. The presence of phosphorus was confirmed by ${ }^{31} \mathrm{P}$ NMR spectrum with Bruker, Switzerland Model AVANCE 400 at $400 \mathrm{MHz}$ using $\mathrm{CDCl}_{3}$ as a solvent and $\mathrm{H}_{3} \mathrm{PO}_{4}$ as an internal standard. Microtrac, Particle size Analyzer USA model was used for Particle size measurement. For particle size study, samples were first diluted with deionized water and subsequently exposed through ultrasonic frequency. The viscosity of flame retardant waterborne polyurethane dispersions (FWBPUDs) was measured at $25{ }^{\circ} \mathrm{C}$ with the spindle 
No.61 at $60 \mathrm{rpm}$ in a Brookfield Digital Viscometer (Model DV-II+PRO). The colloidal stability and appearance determine the safety period for storage of waterborne polyurethane dispersions. This study was examined at room temperature by monitoring any kind of phase separation up to 12 months in sealed, cleaned containers with freshly synthesized FWBPUDs. Thermogravimetric analysis of FWBPU films was performed using Diamond Perkin Elmer Thermogravimetry Analyzer, Pyris-1 TGA model. Samples around 6 to $8 \mathrm{mg}$ were taken and heated from $50^{\circ} \mathrm{C}$ to $500^{\circ} \mathrm{C}$ in a platinum crucible under nitrogen atmosphere at a heating rate of $10^{\circ} \mathrm{C} \mathrm{min}^{-1}$, and the decompositions of samples based on weight loss concerning temperature were recorded. Flame retardant properties of the cured waterborne polyurethane films were determined by limiting oxygen index (LOI) (ASTM D2863) and vertical burning test method Underwriters Laboratories, UL-94 (ASTM D3801). Chemical resistance tests of coated panels were performed according to ASTM D 1647-89.

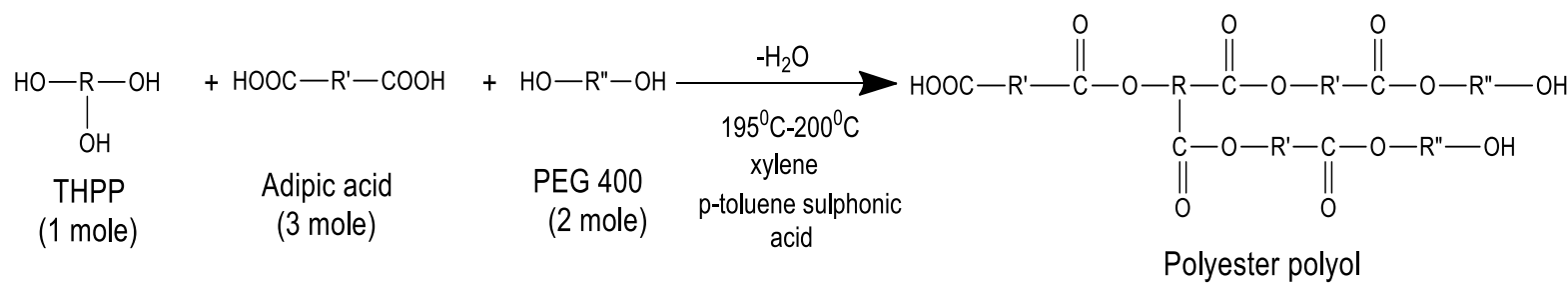

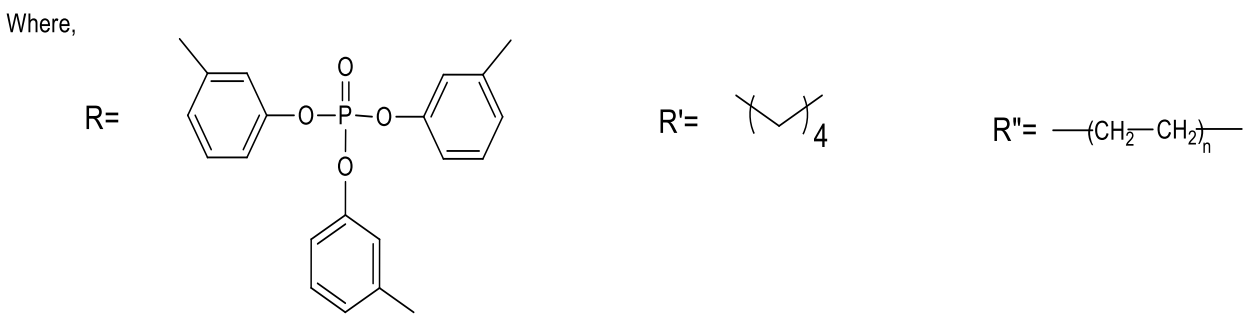

Figure 1. Reaction for the synthesis of polyester-polyol based on phosphorous-containing Tris (mhydroxyphenyl) monophosphate.

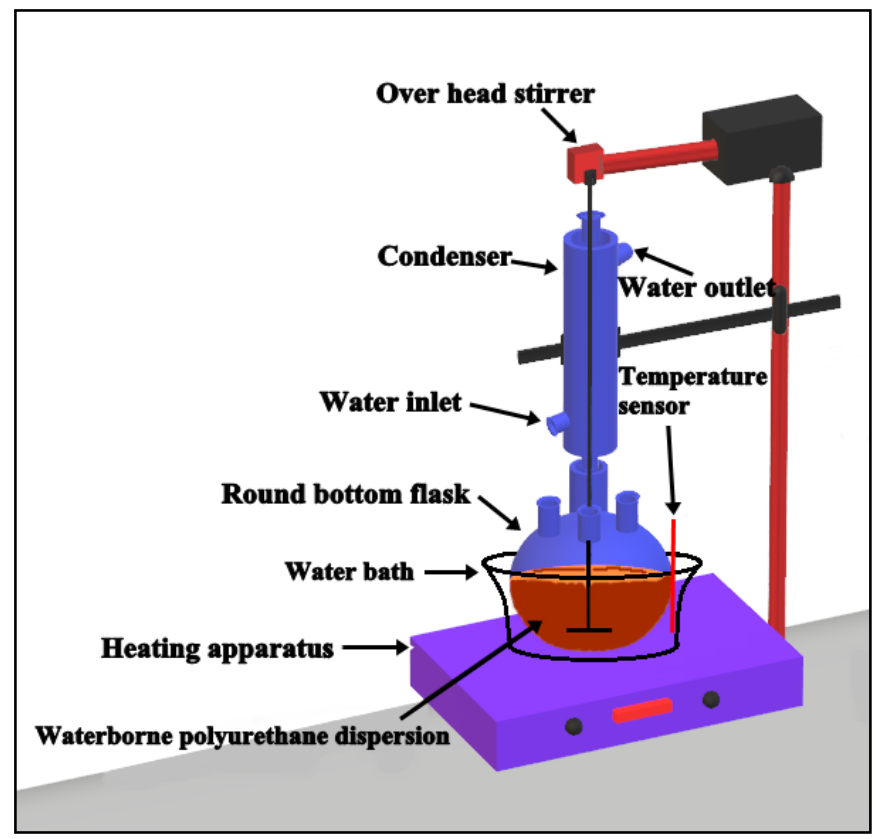

Figure 2. Experimental arrangement for the synthesis of waterborne polyurethane. 


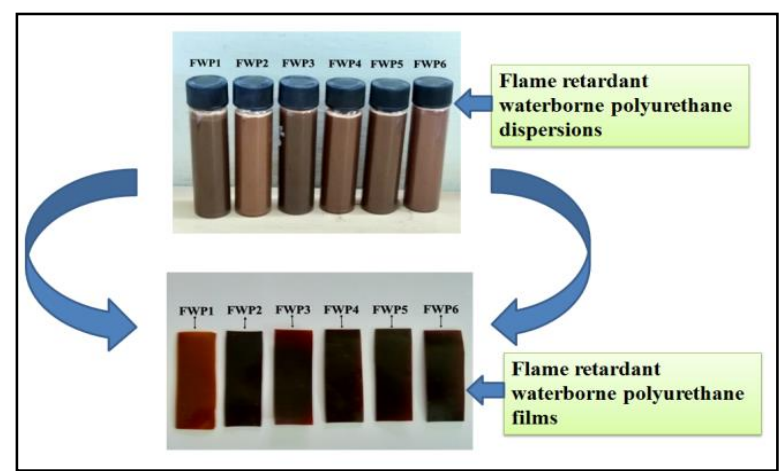

Figure 3. Photographic representation of flame retardant waterborne polyurethane dispersions and films.

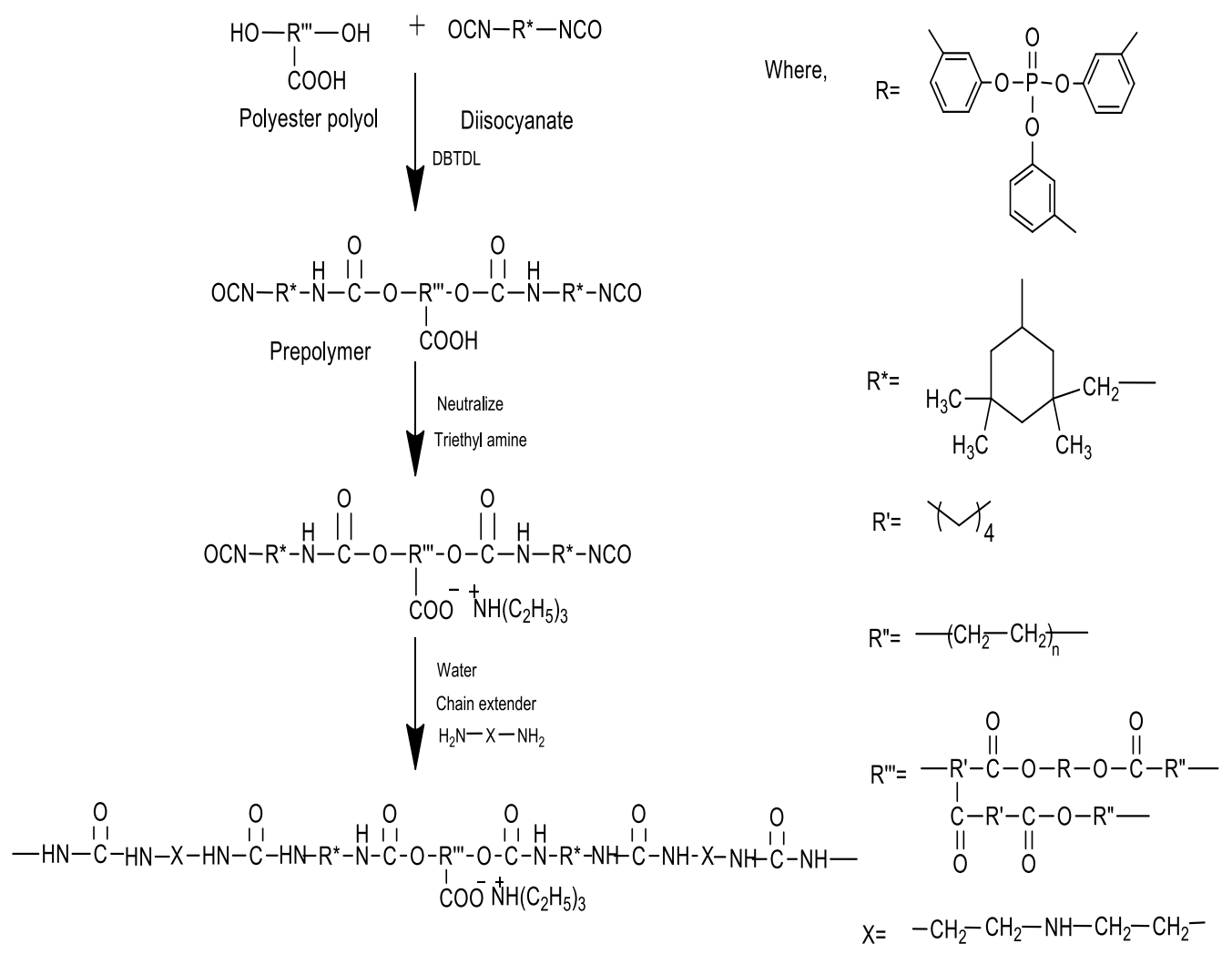

Flame retardant waterborne polyurethane dispersion

Figure 4. Reaction scheme for the synthesis of flame retardant waterborne polyurethane dispersion.

\section{Films and Coatings}

\subsection{Preparation of films.}

Freshly synthesized waterborne polyurethane dispersions were cast onto a Teflon mold and allowed to sit at room temperature over 24 hours. Later, completely tack-free films were kept in the oven at $60^{\circ} \mathrm{C}$ for 8 hours and at $70^{\circ} \mathrm{C}$ for 6 hours. Afterward, cured films were stored in a desiccator at room temperature for further studies.

\subsection{Preparation of coatings.}

Dispersions of waterborne polyurethanes were coated on dirt-free mild steel panels and glass panels with the assistance of a film applicator. Dispersions were allowed to remain on the 
panels for 24 hours at room temperature for setting and were dried out by keeping in an oven at $60^{\circ} \mathrm{C}$ for 8 hours and $70^{\circ} \mathrm{C}$ for 6 hours.

\section{Results and discussion}

\subsection{Characterization of phosphorous-based polyester polyol.}

The number average molecular weight $\bar{M}_{n}$, weight average molecular weight $\bar{M}_{w}$ and polydispersity index as determined through GPC technique is $\bar{M}_{n}=3069, \bar{M}_{w}=4565$ and 1.48 respectively. The acetylation method was used to estimate the number of hydroxyl-free groups in the phosphorus-based polyester polyol. From the estimation, 2 numbers of free hydroxyl groups were obtained.

\subsection{FTIR spectroscopy.}

The FTIR spectrum of polyester-polyol based on phosphorous moiety containing Tris (m-hydroxyphenyl) monophosphate (Figure $5 \mathrm{a}$ ) shows the band at $2869 \mathrm{~cm}^{-1}$ corresponds to $\mathrm{C}-\mathrm{H}$ stretching vibration, the band at $1245 \mathrm{~cm}^{-1}$ is due to the presence of $\mathrm{P}=\mathrm{O}$ group, and band at $1099 \mathrm{~cm}^{-1}$ appears for P-O-C stretching frequency. The band at $1731 \mathrm{~cm}^{-1}$ due to $\mathrm{C}=\mathrm{O}$ ester group gives evidence of the formation of polyester. In Figure $5 \mathrm{~b}$, due to the presence of water in the dispersion systems, the absorption band appears at $3367 \mathrm{~cm}^{-1}$ for (-OH) hydroxyl group. The band at $1600 \mathrm{~cm}^{-1}$ corresponds to $\mathrm{C}=\mathrm{O}$ symmetric stretching frequency, and the band at $1098 \mathrm{~cm}^{-1}$ is due to the P-O-C stretching frequencies. The analysis of FWBPU films (Figure 5 c) shows the absence of a characteristic NCO band at $2270 \mathrm{~cm}^{-1}$ which indicates the formation of urethane linkage [35] as the active hydrogen of a chain extender completely reacted with NCO [5]. N-H stretching band appears at $3392 \mathrm{~cm}^{-1}$. Peaks, respectively, to the absorption of $\mathrm{N}-\mathrm{H}$ and $\mathrm{C}=\mathrm{O}$ groups at $3392 \mathrm{~cm}^{-1}$ and $1730 \mathrm{~cm}^{-1}$ signifies that the FWBPUDs have $-\mathrm{NHCOO}$ (urethane group). The band at $1238 \mathrm{~cm}^{-1}$ is due to the presence of $\mathrm{P}=\mathrm{O}$ group, and the band at $1097 \mathrm{~cm}^{-1}$ is due to the P-O-C stretching frequency. Absorption bands at $2927 \mathrm{~cm}^{-1}$ indicate C$\mathrm{H}$ stretching mode available in the synthesized FWBPUs.

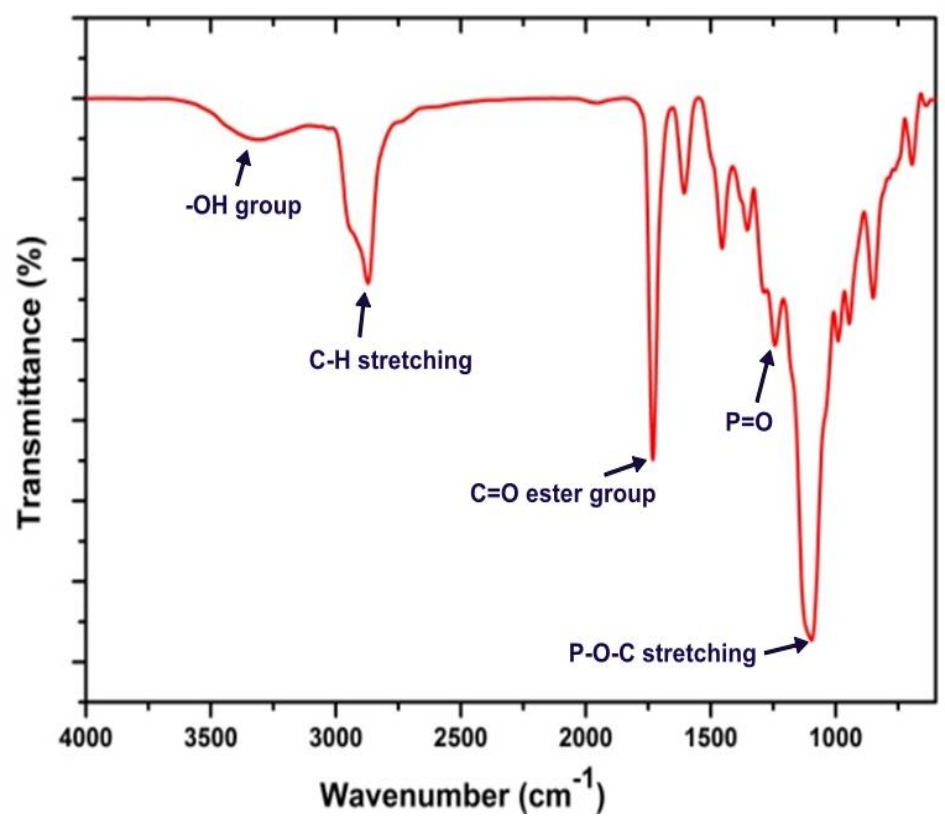

(a) 


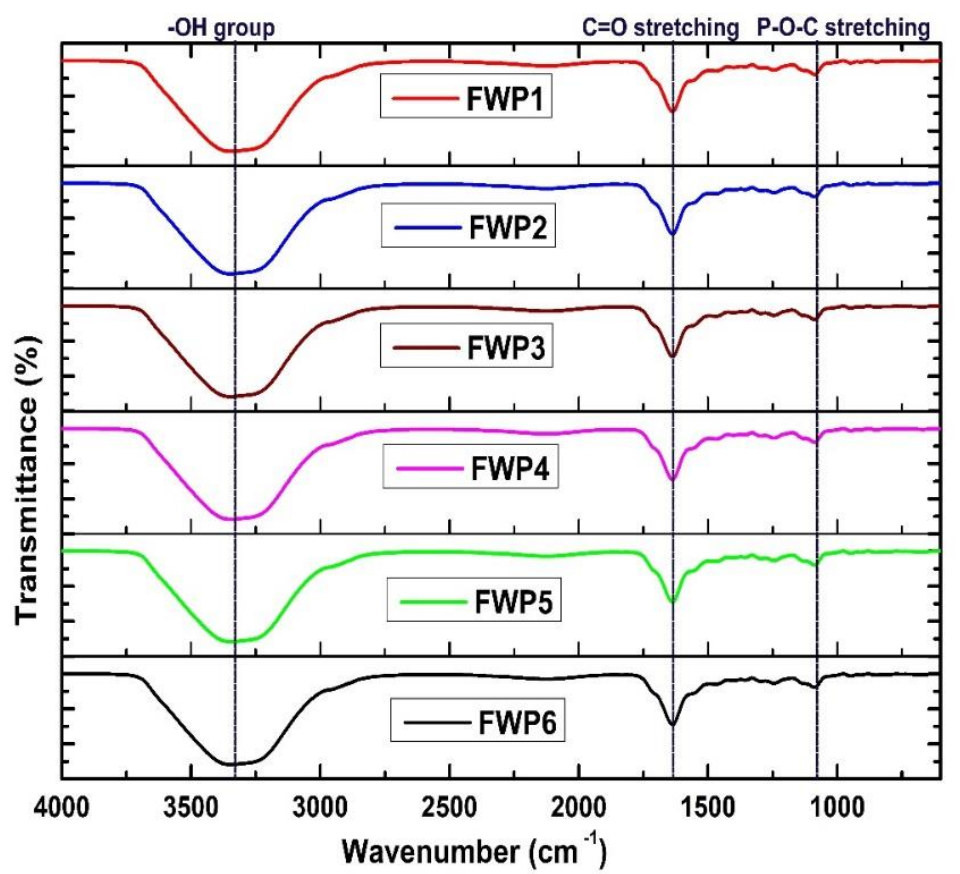

(b)

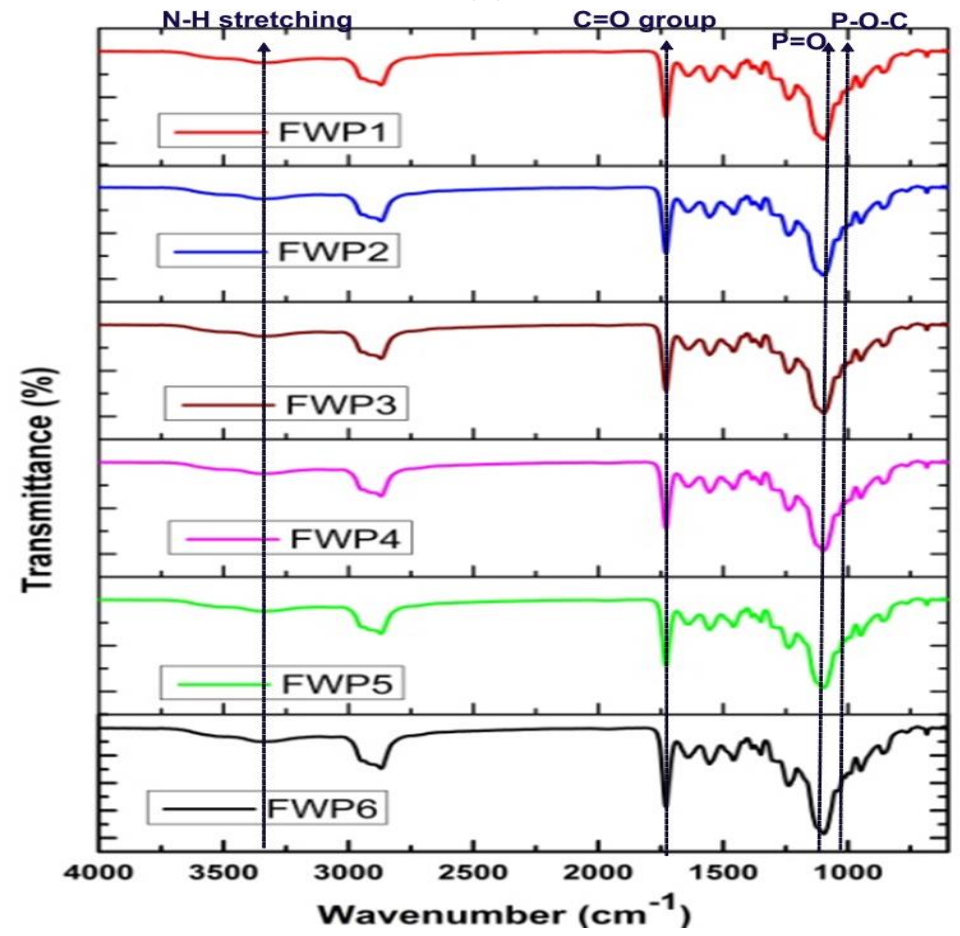

(c)

Figure 5. FTIR spectra of (a) phosphorus-based polyester polyol; (b) six flame-retardant waterborne polyurethane dispersions; (c) six flame-retardant waterborne polyurethane films.

\subsection{NMR spectroscopic analysis.}

Figure 6 clearly indicates the ${ }^{31} \mathrm{P}$ NMR spectrum, which shows a peak at $\delta-17.3 \mathrm{ppm}$ and confirms the presence of phosphorus moiety of Tris (m-hydroxyphenyl) monophosphate in the waterborne polyurethane systems. Thus, phosphorus is present in all the FWBPU systems, although Figure 6 represents the NMR spectrum of one of the system FWBPU systems. 


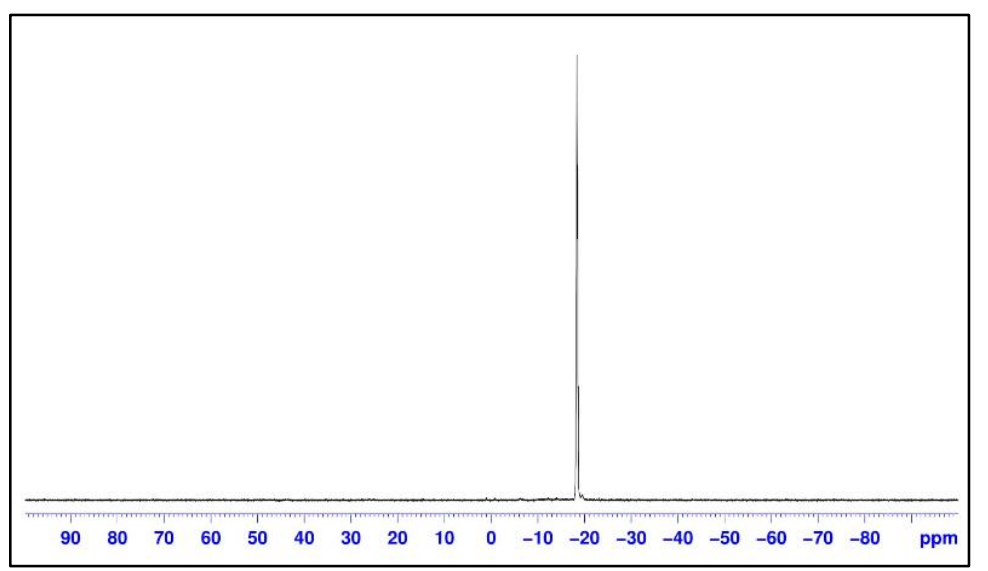

Figure 6. ${ }^{31} \mathrm{P}$ NMR spectrum of flame retardant waterborne polyurethane.

\subsection{Particle size analysis and viscosity measurement.}

The performance of waterborne polyurethane dispersions is highly dependent on the particle size and viscosity of the dispersion systems. The particle size of the WBPUDs are affected due to the change in prepolymer viscosity, $\mathrm{NCO}: \mathrm{OH}$ mole ratio, speed of stirrer, and the method of water addition.

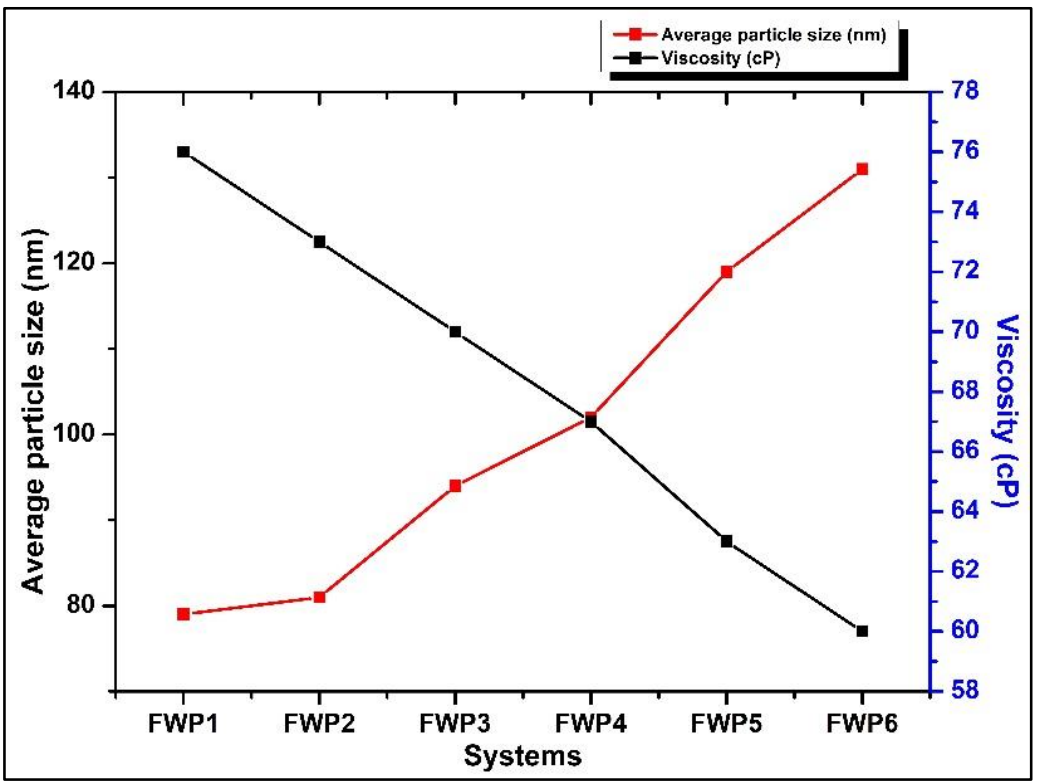

Figure 7. Representation of average particle size and viscosity of FWPUD systems.

The obtained results of particle size, as well as the viscosity of the newly synthesized FWBPUDs, are shown in Figure 7. Data revealed that as the molar ratio of $\mathrm{NCO}: \mathrm{OH}$ increases, the average particle size of WBPUDs is also increasing. As seen in sample FWP1, the average particle size is obtained as $79 \mathrm{~nm}$, where sample FWP6 has an average particle size of $131 \mathrm{~nm}$. This is due to an increment in molar ratio $\mathrm{NCO}: \mathrm{OH}$, whereas the urethane linkage formation increases continuously [36]. As the particle size of dispersions increases, a simultaneous decrement in the viscosity of the WBPUDs is also observed. System FWP1 has $76 \mathrm{cP}$, whereas the lowest viscosity is obtained in sample FWP6, which is $60 \mathrm{cP}$ (Figure 7).

\subsection{Colloidal stability and appearance.}

The safety period for storage and appearance are the important characteristics of waterborne polyurethane dispersions. Freshly synthesized WBPUDs were taken into the clean 
glass containers, which were sealed from the top opening. This study was performed at room temperature, and any kind of phase separation was monitored up to a period of 12 months. The result of the inspections is shown in Table 1. The appearance showed that the color of dispersions did not vary with the change in a molar ratio of $\mathrm{NCO}: \mathrm{OH}$ groups. Hence, these systems and their formulations gave least or no discoloration during and after the production of dispersions. The color of the final waterborne polyurethane systems is governed by the color of different reagents used during the synthesis, i.e., the color of the base product (monomer), the color of polyester polyol, and diisocyanate. After 8 months, precipitates were detected in FWP6 system. The reason is as the mole ratio of $\mathrm{NCO}: \mathrm{OH}$ was increased, the average particle size of the FWP6 became maximum among all the systems due to the agglomeration of the particles. Hence in the FWP6 system, precipitates were appeared earlier as compared to that of the other systems.

Table 1. Colloidal stability and appearance of different waterborne polyurethane systems.

\begin{tabular}{l|l|l|l} 
Systems & $\begin{array}{l}\text { Mole ratio } \\
\text { NCO:OH }\end{array}$ & Colloidal stability & Appearance \\
\hline FWP1 & $1.5: 1$ & $>9$ months & Brown \\
\hline FWP2 & $1.6: 1$ & $>9$ months & Brown \\
\hline FWP3 & $1.7: 1$ & $>9$ months & Brown \\
\hline FWP4 & $1.8: 1$ & $>9$ months & Brown \\
\hline FWP5 & $1.9: 1$ & $>9$ months & Brown \\
\hline FWP6 & $2.0: 1$ & $>8$ months & Brown
\end{tabular}

\subsection{Properties of casted films.}

\subsubsection{Flame retardant properties.}

Table 2 shows the flame retardant properties like LOI and UL-94 of the films. The LOI is a measurement of the minimal oxygen concentration in a mixture of oxygen and nitrogen that will merely support flaming combustion of material under specified conditions [37,38]. To achieve self-extinguishing property, the material LOI value should be greater than $26 \%$. This provides a measure of the ease of extinction of the materials. This is an appropriate method for a semi-qualitative indicator of the efficiency of flame retardant polyurethanes. This is because the equipment is low-cost, and the sample size requirement is less [39]. In LOI test, it is evaluated that the sample FWP1 with the minimum content of NCO:OH having the smallest particle size, and the less number of urethane linkages started to burn at $29 \%$ LOI value. For sample FWP4, a little hike is observed than for the rest of the systems. For FWP5 and FWP6, the value of LOI is remaining constant, i.e., 32. This result signifies that there is no proportional increase in flame retardancy with an increasing molar ratio of diisocyanate. In UL-94, the test of all the samples, $V_{0}$ rating is attained, which denotes that for the vertical samples under observation, the burning stops within 10 seconds [40]. None of the test samples burnt longer than 10 seconds, and no burnt particles were observed during or at the end of the test. These observations show that all the samples have good flame retardancy. Experimental arrangements for the UL-94 V test and LOI flammability test are displayed in Figure 8 and Figure 9, respectively. In addition to this, Figure 10 shows the photographic representation of LOI testing for the FWBPU film. 
Table 2. Flame retardant properties of different WBPUDs systems

\begin{tabular}{l|l|l|l} 
Systems & $\begin{array}{l}\text { Mole ratio } \\
\text { NCO:OH }\end{array}$ & LOI (\%) & UL-94 \\
\hline FWP1 & $1.5: 1$ & 29 & $\mathrm{~V}_{0}$ \\
\hline FWP2 & $1.6: 1$ & 29 & $\mathrm{~V}_{0}$ \\
\hline FWP3 & $1.7: 1$ & 30 & $\mathrm{~V}_{0}$ \\
\hline FWP4 & $1.8: 1$ & 31 & $\mathrm{~V}_{0}$ \\
\hline FWP5 & $1.9: 1$ & 32 & $\mathrm{~V}_{0}$ \\
\hline FWP6 & $2.0: 1$ & 32 & $\mathrm{~V}_{0}$ \\
\hline
\end{tabular}

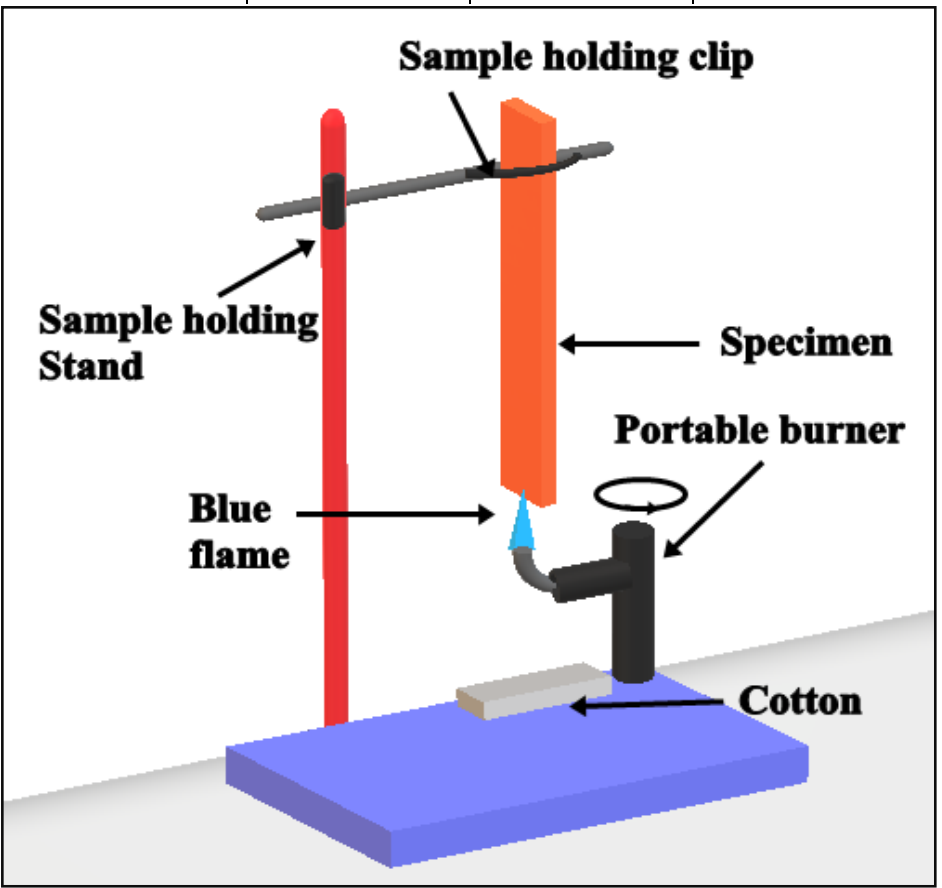

Figure 8. Experimental arrangement for the UL-94 V test.

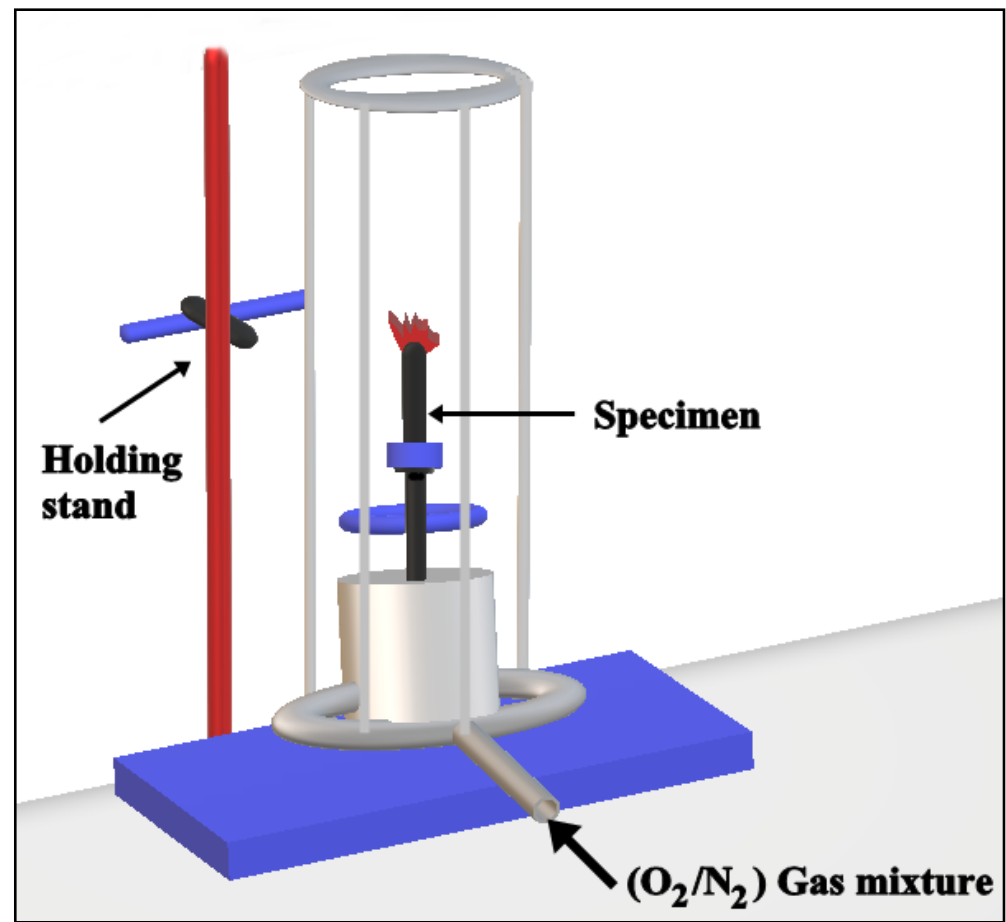

Figure 9. Experimental arrangement for the LOI flammability test. 


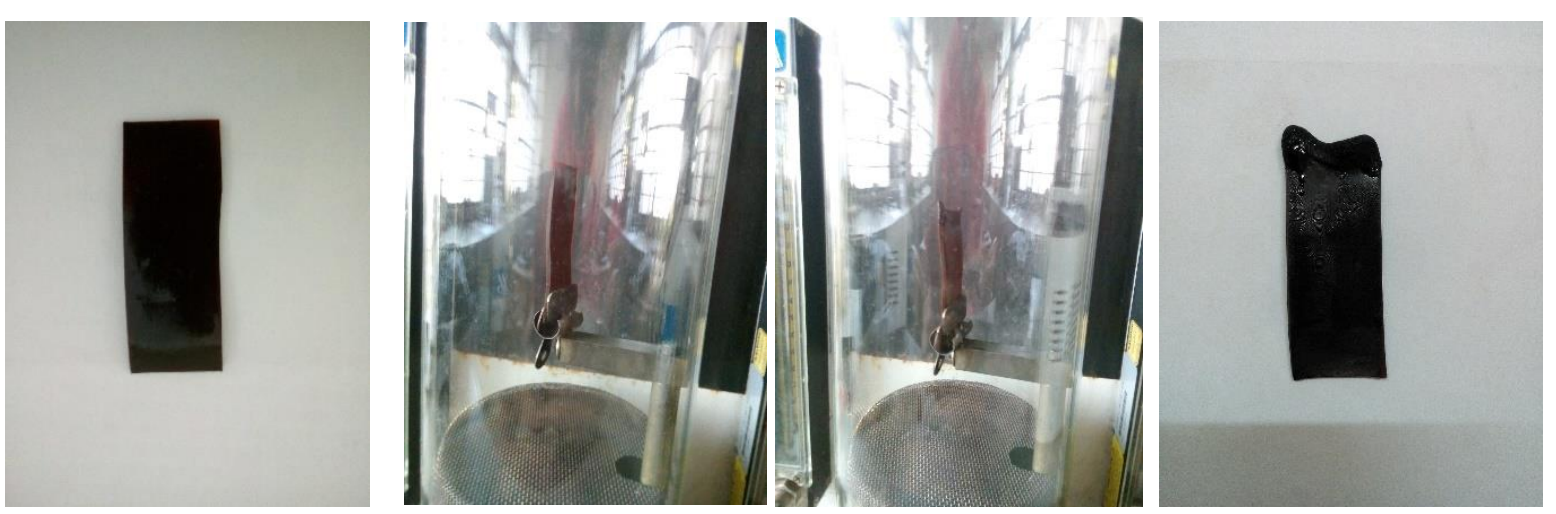

Figure 10. LOI test performance (a) FWBPU film sample; (b) Sample kept in the LOI testing apparatus before the analysis; (c) After completion of LOI test; (d) sample removed from the LOI testing apparatus.

\subsubsection{Thermogravimetric analysis (TGA).}

Thermogravimetric analysis was done to determine thermal stability and percentage char yield of the cured waterborne polyurethane films. Figure 11 represents the characteristics thermograms, and in Table 3, the data are reported. The results from the analysis show that the thermal stability of FWP6 is highest, and for the same system, char formation is also maximum among all the systems, which is $14.13 \%$. In the FWP5 system, the char value obtained is less than that of FWP6, but at $70 \%$ weight loss, both the systems are stable at $400^{\circ} \mathrm{C}$, which is also a significant temperature of both the systems. In the cured films of WBPUs, considerable heat energy is required to break bonds inside the bulk polymeric matrix. For that reason, the thermal decomposition of cured WBPU films starts preferentially through side chains. The decomposition process of WBPU is a complex matter involving chemical and physical phenomena which are not quantized to a single process. With the increasing thermal perturbance, the bonds in the polymer molecules undergo thermal agitation, rotation or vibration of polymeric fragments fixed to local space. On further increment in the heat energy, the bonds tend to break and form multiple radical fragments. Formation of smaller molecules is also probable, followed by vaporization or diffuse out. The final decomposition step involves removing all the volatiles, and the process leaves a char at the end of the decomposition. Char being insoluble and containing a major amount of carbon does not further change the phase, and hence a rigid and stable phase is obtained, which cuts down the oxygen supply, and combustion is then least probable [41-43].

During the analysis, the strongest interactions of hydrogen bonds with urethanes, and urea moieties provide high thermal stability and the highest char yield with respect to increasing the NCO:OH ratio of the systems [44]. Hence it concludes that the higher the mole ratio of $\mathrm{NCO}: \mathrm{OH}$, the superior is the enrichment in cross-linking density which ensures high thermal stability [45]. For all the samples, $10 \%$ weight loss occurs in the temperature range of $300^{\circ} \mathrm{C}-316^{\circ} \mathrm{C}$. This may correspond to the breakage of the urethane bonds, which leads to the formation of carbon dioxide, alcohols, amines, carbon monoxide, etc. In the range of $366^{\circ} \mathrm{C}$ $371^{\circ} \mathrm{C}$, a weight loss of $30 \%$ is seen, which is due to the decomposition of ester links followed by the formation of char which starts around $420^{\circ} \mathrm{C}$. At $500^{\circ} \mathrm{C}$, the final char values are obtained when the stable weight observed in the thermogram corresponding to no further weight changes. 
Table 3. Thermal properties of waterborne polyurethane films.

\begin{tabular}{|c|c|c|c|c|c|}
\hline \multirow[t]{2}{*}{ Systems } & \multirow{2}{*}{$\begin{array}{l}\text { Mole ratio } \\
\text { NCO:OH }\end{array}$} & \multicolumn{3}{|c|}{$\%$ Weight loss from TGA in nitrogen $\left({ }^{\circ} \mathrm{C}\right)$} & \multirow{2}{*}{$\begin{array}{l}\text { Char yield at } \\
500^{\circ} \mathrm{C}(\%)\end{array}$} \\
\hline & & $10 \%$ & $30 \%$ & $70 \%$ & \\
\hline FWP1 & $1.5: 1$ & 316 & 364 & 398 & 11.29 \\
\hline FWP2 & $1.6: 1$ & 304 & 368 & 399 & 11.63 \\
\hline FWP3 & $1.7: 1$ & 308 & 369 & 399 & 12.19 \\
\hline FWP4 & $1.8: 1$ & 308 & 371 & 400 & 12.54 \\
\hline FWP5 & 1.9:1 & 300 & 366 & 400 & 12.74 \\
\hline FWP6 & $2.0: 1$ & 308 & 369 & 400 & 14.13 \\
\hline
\end{tabular}

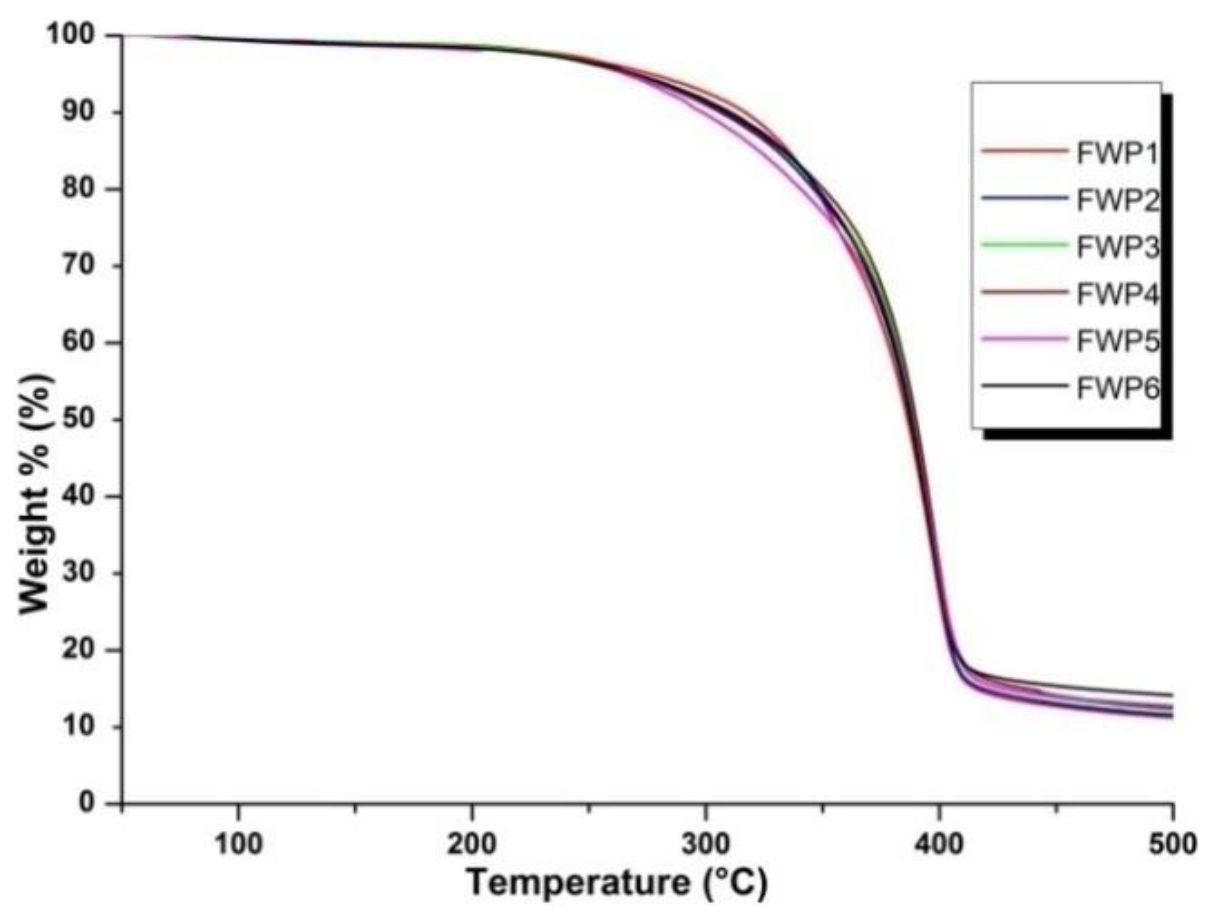

Figure 11. TGA thermograms of flame retardant waterborne polyurethanes at the heating rate of $10^{\circ} \mathrm{C} / \mathrm{min}$.

\subsection{Properties of coatings.}

5.7.1. Mechanical properties of the coated panels according to ASTM standards.

Mechanical properties of WBPUDs of coated panels like pencil hardness, scratch resistance, adhesion (cross-hatch), flexibility 1/4" mandrel are determined according to ASTM standard and are shown in Table 4. Pencil hardness data explains that the hardness increases with the increment of NCO:OH molar ratio for the first three systems, i.e., FWP1, FWP2, and FWP3 but its leftovers same for the other three systems, specifically FWP4, FWP5, and FWB6. Scratch hardness is the most significant test mandatory to determine the properties of coatings [46]. A scratch hardness tester was used to determine the hardness (ASTM D 5178) of the coatings, which exhibits the durability of the coatings. For different compositions, i.e., varying $\mathrm{NCO}: \mathrm{OH}$ mole ratio, the scratch hardness of the coated panels is found to be different. Scratch hardness increases as the $\mathrm{NCO}: \mathrm{OH}$ ratios increase due to the increment in the hard segments in the polymer due to the increment in the urethane linkages. Adhesion (ASTM D 3359) and flexibility (ASTM D 522) are the principal characteristics of all coatings. The results of flexibility and adhesion are shown in Table 4. These tests reveal that all the systems pass through scrutiny. 
Table 4. Mechanical properties of waterborne polyurethane coatings.

\begin{tabular}{l|l|l|l|l|l} 
Systems & $\begin{array}{l}\text { Mole ratio } \\
\text { NCO:OH }\end{array}$ & Pencil hardness & $\begin{array}{l}\text { Scratch } \\
\text { resistance } \\
\text { Gms }\end{array}$ & $\begin{array}{l}\text { Adhesion } \\
\text { (cross-hatch) }\end{array}$ & $\begin{array}{l}\text { Flexibility } \\
\mathbf{1 / 4} \text { " mandrel }\end{array}$ \\
\hline FWP1 & $1.5: 1$ & $1 \mathrm{H}$ & 1780 & PASS & PASS \\
\hline FWP2 & $1.6: 1$ & $2 \mathrm{H}$ & 2175 & PASS & PASS \\
\hline FWP3 & $1.7: 1$ & $3 \mathrm{H}$ & 2230 & PASS & PASS \\
\hline FWP4 & $1.8: 1$ & $4 \mathrm{H}$ & 2400 & PASS & PASS \\
\hline FWP5 & $1.9: 1$ & $4 \mathrm{H}$ & 2655 & PASS & PASS \\
\hline FWP6 & $2.0: 1$ & $4 \mathrm{H}$ & 2680 & PASS \\
& $* 6 \mathrm{H}>5 \mathrm{H}>4 \mathrm{H}>3 \mathrm{H}>2 \mathrm{H}>1 \mathrm{H}>\mathrm{H}>1 \mathrm{HB}>2 \mathrm{HB}>3 \mathrm{HB}>4 \mathrm{HB}>5 \mathrm{HB}>6 \mathrm{HB}$.
\end{tabular}

5.7.2. Chemical resistance properties of coated panels.

The outcome of the chemical and solvent resistance properties of coated panels portrayed in Table 5 are optimistic to a certain extent in cured coating performance. Chemical resistance tests of coated panels were carried out in accordance with ASTM D 1647-89 by immersing the panels in various mediums, i.e., water, acid, methanol, and alkali solutions. All the corners of the glass panel were sealed with wax. This was done to prevent any leakage from the glass panel's corners. Water, methanol, 3\% (w/w) $\mathrm{HCl}$ solution and 3\% (w/w) $\mathrm{NaOH}$ solution were used to dip the prepared panels. After 10 days, the samples were inspected for visual appearance. Due to lower cross-linking density of lower mole ratio of NCO:OH, FWP1 and FWP2 show discoloration in methanol as well as in alkali, and there appeared a softening of coating in the acid test. While, in FWP3, discoloration was observed in the acid test. Acid and alkali resistance of the panels based on high $\mathrm{NCO}: \mathrm{OH}$ mole ratio showed better chemical resistance. In a lower molar ratio of 1.5:1.0 and 1.6:1.0, the chemicals can easily penetrate into the polyurethane layer due to the lower number of urethane linkages present compared to a higher amount of NCO:OH mole ratios[47-52]. All the coatings were stable; no cases of removal of the coating were observed in the case of water. Cracking was not observed in any case.

Table 5. Chemical resistance properties of WBPUs coated on panels.

\begin{tabular}{l|l|c|c|c|c} 
System & $\begin{array}{l}\text { Mole ratio } \\
\text { NCO:OH }\end{array}$ & Water & Methanol & $\begin{array}{c}\text { Alkali } \\
\text { resistance } \\
\mathbf{3 \%} \\
\text { NaOH }\end{array}$ & $\begin{array}{c}\text { Acid } \\
\text { resistance } \\
\mathbf{3 \%} \\
\text { HCl }\end{array}$ \\
\hline FWP1 & $1.5: 1$ & 5 & 4 & 4 & 3 \\
\hline FWP2 & $1.6: 1$ & 5 & 4 & 4 & 3 \\
\hline FWP3 & $1.7: 1$ & 5 & 5 & 5 & 4 \\
\hline FWP4 & $1.8: 1$ & 5 & 5 & 5 & 5 \\
\hline FWP5 & $1.9: 1$ & 5 & 5 & 5 & 5 \\
\hline FWP6 & $2.0: 1$ & 5 & 5 & 5 & 5
\end{tabular}

1- coating completely removed, 2-Cracking appears, 3-Softening, 4 - Discoloration, 5- No change.

\section{Conclusions}

In the present study, the synthesized product governs the dependability of various physicochemical properties on the NCO:OH ratio. FTIR spectra disclose the characteristic absorption bands of urethane, confirming the formation of waterborne polyurethane and the 
absence of -NCO peak, which confirms all the systems are eco-friendly. TGA analysis shows that the relatively higher thermal stability and \% char yield are observed in the higher NCO:OH mole ratio samples. Significant flame retardant property is observed by incorporating phosphorous moiety in the polymeric chain. LOI and UL-94 results show appreciable flame retardancy. The increment of diisocyanate content in the flame retardant waterborne polyurethane systems makes no change in LOI value. In the particular case of the pencil hardness test, the value of $4 \mathrm{H}$ is observed, which is appreciable in terms of coating property. Scratch hardness continuously increases with varying NCO:OH mole ratios because of the increment in the hard segments in the polymer, resulting in an increment in the urethane linkages in the respective systems. The chemical resistance properties are exceptionally good. All the systems are extremely good and pass through the flexibility and adhesion tests. Therefore, the coating and casting results shed new light on the utility of these synthesized flame retardant waterborne polyurethanes systems to a vast level.

\section{Funding}

This research received no external funding.

\section{Acknowledgments}

We would like to thank the Department of Materials Science, Sardar Patel University, for providing us advanced laboratory and necessary instrumental facilities to complete this research study.

\section{Conflicts of Interest}

The authors declare no conflict of interest.

\section{References}

1. Kim, C.K.; Kim, B. K. IPDI based polyurethane ionomer dispersions: effects of ionic, nonionic hydrophilic segments, and extender on particle size and physical properties of emulsion cast film. J. Appl. Polym. sci. 1991, 43, 2295-2301, https://doi.org/10.1002/app.1991.070431219.

2. Shao, C.H.; Huang, J.J.; Chen, G.N.; Yeh, J.T.; Chen, K.N. Thermal and combustion behaviors of aqueousbased polyurethane system with phosphorus and nitrogen containing curing agent. Polym. Degrad. Stab. 1999, 65, 359-371, https://doi.org/10.1016/S0141-3910(99)00025-7.

3. Zhang, C.; Liang, H.; Liang, D.; Lin, Z.; Chen, Q.; Feng, P.; Wang, Q. Renewable Castor-Oil-based Waterborne Polyurethane Networks: Simultaneously Showing High Strength, Self-Healing, Processability and Tunable Multishape Memory. Angew. Chem. Int. Ed. 2021. 60, 8, 4289-4299, https://doi.org/10.1002/anie.202014299.

4. Dai, M.; Zhai, Y.; Zhang, Y. A green approach to preparing hydrophobic, electrically conductive textiles based on waterborne polyurethane for electromagnetic interference shielding with low reflectivity. Chem. Eng. J. 2021, 421, 127749, https://doi.org/10.1016/j.cej.2020.127749.

5. Santamaria-Echart, A.; Fernandes, I.; Barreiro, F.; Corcuera, M. A.; Eceiza, A. Advances in waterborne polyurethane and polyurethane-urea dispersions and their eco-friendly derivatives: A review. Polym. 2021, 13,3, 409, https://doi.org/10.3390/polym13030409.

6. Coutinho, F.M.B.; Delpech, M.C. Some properties of films cast from polyurethane aqueous dispersions of polyether-based anionomer extended with hydrazine. Polym. Test. 1996, 15, 103-113, https://doi.org/10.1016/0142-9418(95)00016-X.

7. Coutinho, F.M.B.; Delpech, M.C. Degradation profile of films cast from aqueous polyurethane dispersions. Polym. Degrad. Stab. 2000, 70, 49-57, https://doi.org/10.1016/S0141-3910(00)00087-2. 
8. Zhou, W.; Gong, X.; Li, Y.; Si, Y.; Zhang, S.; Yu, J.; Ding, B. Environmentally friendly waterborne polyurethane nanofibrous membranes by emulsion electrospinning for waterproof and breathable textiles. Chem. Eng. J. 2021,130925, https://doi.org/10.1016/j.cej.2021.130925.

9. Liang, Z.; Zhu, J.; Li, F.; Wu, Z.; Liu, Y.; Xiong, D. Synthesis and properties of self-crosslinking waterborne polyurethane with side chain for water-based varnish. Prog. Org. Coat. 2021, 150, 105972, https://doi.org/10.1016/j.porgcoat.2020.105972.

10. Agnol, L. D.; Fernanda T.G.D.; Heitor L.O.; Marco S.; Otavio B. UV-curable waterborne polyurethane coatings: A state-of-the-art and recent advances review. Prog. Org. Coat. 2021, 154, 106156, https://doi.org/10.1016/j.porgcoat.2021.106156.

11. Çelebi, F.; Aras, L.; Gündüz, G.; Akhmedov, I.M. Synthesis and Characterization Of Waterborne and Phosphorus-Containing Flame Retardant Polyurethane Coatings. J. Coat. Technol. 2003, 75, 65-71, https://doi.org/10.1007/BF02757863.

12. Madbouly, S. A. Waterborne Polyurethane Dispersions and Thin Films: Biodegradation and Antimicrobial Behaviors. Molecules, 2021, 26,4, 961, https://doi.org/10.3390/molecules26040961.

13. Chen, S.Y.; Ren-Quan Z.; Fu-Sheng C.; Syang-Peng R. Synthetic scheme to increase the abrasion resistance of waterborne polyurethane-urea by controlling micro-phase separation. J. Appl. Polym. sci. 2021, 138, 24, 50561, https://doi.org/10.1002/app.50561.

14. Zhang, X.; Cai, Y.; Zhang, X.; Aziz, T.; Fan, H.; Bittencourt, C. Synthesis and characterization of eugenolbased silicone modified waterborne polyurethane with excellent properties. J. Appl. Polym. sci. 2021, 138, 22, 50515, https://doi.org/10.1002/app.50515.

15. Zhang, P.; Yazhou, H.; Saiqi, T.; Haojun, F.; Yi, C.; Yan, J. Flame retardancy, mechanical, and thermal properties of waterborne polyurethane conjugated with a novel phosphorous-nitrogen intumescent flame retardant. Polym. Compo. 2017, 38, 452-462, https://doi.org/10.1002/pc.23603.

16. Çelebi, F.; Osman, P.; Leyla, A.; Güngör, G.; Akhmedov, I.M. Synthesis and characterization of waterdispersed flame-retardant polyurethane resin using phosphorus-containing chain extender. J. Appl. Polym. Sci. 2004, 91, 1314-1321.

17. Shaolin, L.; Yechang F.; Peikun Z.; Wei H.; Yi C.; Haojun F.; Dingshan Y.; Xudong C. Preparation of FlameRetardant Polyurethane and Its Applications in the Leather Industry. Polym. 2021, 13, 11, 1730. https://doi.org/10.3390/polym13111730.

18. Kuan, H.C.; Chi, M.M.C.; Ping, C.W. Y.; Ming, S.; Ho, W.H.; Lee, T.M. Synthesis, thermal, mechanical and rheological properties of multiwall carbon nanotube/waterborne polyurethane nanocomposite. Compo. Sci. Technol. 2005, 65, 11-12, 1703-1710, https://doi.org/10.1016/j.compscitech.2005.02.017.

19. Pathak, S.S.; Sharma, A.; Khanna, A. S. Value addition to waterborne polyurethane resin by silicone modification for developing high performance coating on aluminum alloy. Prog. Org. Coat. 2009, 65, 206216, https://doi.org/10.1016/j.porgcoat.2008.11.005.

20. Zhu, H. P.; Zumao, C.; Yongmei, L.; Gaiyun, W.; Lei, T.; Yang, P.; Ran, Z. U. H.; Khan, P. W. Preparation and characterization of flame retardant polyurethane foams containing phosphorus-nitrogen-functionalized lignin. RSC Advances. 2014, 4, 55271-55279, https://doi.org/10.1039/C4RA08429B.

21. Lu, S.Y.; Hamerton, I. Recent developments in the chemistry of halogen-free flame retardant polymers, Prog. Polym. Sci. 2002, 27, 1661-1712.

22. D.K. Chattopadhyay, D.C. Webster, Thermal stability and flame retardancy of polyurethanes. Prog. Polym. Sci. 2009, 34, 1068-1133.

23. T.C. Chang, Y.S. Chiu, H.B. Chen, S.Y. Ho, Degradation of phosphorus-containing polyurethanes, Polym. Degrad. Stab. 1995, 47, 375-381, https://doi.org/10.1016/S0079-6700(02)00018-7.

24. Kim, B. K. Aqueous polyurethane dispersions. Colloid. Polym. Sci. 1996, 274, 599-611, https://doi.org/10.1007/BF00653056.

25. Dai, Z.; Jiang, P.; Lou, W.; Zhang, P.; Bao, Y.; Gao, X.; Xia, J.; Haryono, A. Preparation of degradable vegetable oil-based waterborne polyurethane with tunable mechanical and thermal properties. Eur. Polym. J. 2020, 139, 109994, https://doi.org/10.1016/j.eurpolymj.2020.109994.

26. Liu, Q.; Liao, B.; Pang, H.; Lu, M.; Meng, Y. Preparation and characterization of a self-matting coating based on waterborne polyurethane-polyacrylate hybrid dispersions. Prog. Org. Coat. 2020, 143, 105551, https://doi.org/10.1016/j.porgcoat.2020.105551.

27. Yang, Z.; Wu, G. Effects of soft segment characteristics on the properties of biodegradable amphiphilic waterborne polyurethane prepared by a green process. J. Mater. Sci. 2020, 55,7, 3139-3156, https://doi.org/10.1007/s10853-019-04237-6. 
28. Hu, L.; Pu, Z.; Zhong, Y.; Liu, L.; Cheng, J.; Zhong, J. Effect of different carboxylic acid group contents on microstructure and properties of waterborne polyurethane dispersions. J. Polym. Res. 2020, 27, 5, 1-9, https://doi.org/10.1007/s10965-020-02125-1.

29. Noble, K.L. Waterborne polyurethanes. Prog. Org. Coat. 1997, 32, 131-136, https://doi.org/10.1016/S03009440(97)00071-4.

30. Sardon, H.; Lourdes, I.; José, F.B.M.; Luna, J.; Lansalot, M.; Elodie, B.L. Waterborne polyurethane dispersions obtained by the acetone process: A study of colloidal features. J. Appl. Polym. Sci. 2011, 120, 2054-2062, https://doi.org/10.1002/app.33308.

31. Subramani, S.; Park, Y.J.; Lee, Y.S.; Kim, J.H. New development of polyurethane dispersion derived from blocked aromatic diisocyanate. Prog. Org. Coat. 2003, 48, 71-79, https://doi.org/10.1016/S03009440(03)00118-8.

32. Patel, R. H.; Shah, M. D.; Patel, H. B. Synthesis and characterization of structurally modified polyurethanes based on castor oil and phosphorus-containing polyol for flame-retardant coatings. Int. J. Polym. Anal. Character. 2011, 16, 2, 107-117, https://doi.org/10.1080/1023666X.2011.541108.

33. Nanda, A.K.; Wicks, D.A. The influence of the ionic concentration, concentration of the polymer, degree of neutralization and chain extension on aqueous polyurethane dispersions prepared by the acetone process. Polym. 2006, 47, 1805-1811, https://doi.org/10.1016/j.polymer.2006.01.074.

34. Patel, R.H.; Kapatel, P.M. Studies on the effect of the size of waterborne polyurethane nanoparticles on properties and performance of coatings. Int. J. Polym. Anal. Character. 2018, 1, 1-9.

35. Lu, M.G.; Lee, J.Y.; Shim, M.J.; Kim, S.W. Synthesis and properties of anionic aqueous polyurethane dispersions. J. Appl. Polym. Sci. 2002, 86, 3461-3465, https://doi.org/10.1002/app.10770.

36. Patel, R.H.; Patel, K.S. Synthesis and characterization of flame retardant hyperbranched polyurethanes for nano-composite and nano-coating applications. Prog. Org. Coat. 2015, 88, 283-292, https://doi.org/10.1016/j.porgcoat.2015.07.007.

37. Weil, E. D., Levchik, S. Current practice and recent commercial developments in flame retardancy of polyamides. J. Fire. Sci. 2004, 22, 251-264, https://doi.org/10.1177/0734904104040546.

38. Lu, S.Y.; Hamerton, I. Recent developments in the chemistry of halogen-free flame retardant polymers. Prog. Polym. Sci. 2002, 27, 1661-1712, https://doi.org/10.1016/S0079-6700(02)00018-7.

39. Laoutid, F.; Bonnaud, L.; Alexandre, M.; Cuesta, J.M.L.; Dubois, P.h. New prospects in flame retardant polymer materials: from fundamentals to nanocomposites. Mat. Sci. Eng. R. 2009, 63, 100-125, https://doi.org/10.1016/j.mser.2008.09.002.

40. Chattopadhyay, D.K.; Raju, K. V. S. N. Structural engineering of polyurethane coatings for high performance applications. Prog. Polym. Sci. 2007, 32, 352-418, https://doi.org/10.1016/j.progpolymsci.2006.05.003.

41. Zhang, Y.; Zhengbin, X.; Hong, H.; Chen, H. A degradation study of waterborne polyurethane based on TDI. Polym. Test. 2009, 28, 264-269, https://doi.org/10.1016/j.polymertesting.2008.12.011.

42. Patel, R.; Kapatel, P. Waterborne polyurethanes: A three step synthetic approach towards environmental friendly flame retardant coatings. Prog. Org. Coat. 2018, 125, 186-194, https://doi.org/10.1016/j.porgcoat.2018.09.010.

43. Sergei, S.V.; Edward, D.W. Thermal decomposition, combustion and fire retardancy of polyurethanes-a review of the recent literature. Polym. Int. 2004, 53, 1585-1610, https://doi.org/10.1002/pi.1314.

44. Bosco, J.V.; Balasubramaniyan, N. Waterborne polyurethane from polycaprolactone and tetramethylxylene diisocyanate: Synthesis by varying $\mathrm{NCO} / \mathrm{OH}$ ratio and its characterization as wood coatings. Open J. Org. Polym. Mater. 2013, 4,1, 37, https://doi.org/10.4236/ojopm.2014.41006.

45. Sørensen, P. A.; Kiil, S.; Dam-Johansen, K.; Weinell, C. E. Anticorrosive coatings: a review. J. Coat. Technol. Res. 2009, 6, 2, 135-176, https://doi.org/10.1007/s11998-008-9144-2.

46. Dave, D.; Mestry, S.; Mhaske, S. T. Development of flame-retardant waterborne polyurethane dispersions (WPUDs) from sulfonated phosphorus-based reactive water-dispersible agents. J. Coat. Technol. Res. 2021, 1-13, https://doi.org/10.1007/s11998-020-00458-6.

47. Wang, C.; Zhang, J.; Huang, J.; Wang, H.; He, M.; Ding, L. Flame Retardant Modified Bio-Based Waterborne Polyurethane Dispersions Derived from Castor Oil and Soy Polyol. Eur. J. Lipid. Sci. Technol. 2021, 123, 2000248, https://doi.org/10.1002/ejlt.202000248.

48. Wang, S.; Du, X.; Fu, X.; Du, Z.; Wang, H.; Cheng, X. Highly effective flame-retarded polyester diol with synergistic effects for waterborne polyurethane application. J. Appl. Polym. sci. 2020, 137, 48444, https://doi.org/10.1002/app.48444. 
49. Jang, T.; Kim, H. J.; Jang, J. B.; Kim, T. H.; Lee, W.; Seo, B.; Lim, C. S. Synthesis of waterborne polyurethane using phosphorus-modified rigid polyol and its physical properties. Polym. 2021, 13, 432, https://doi.org/10.3390/polym13030432.

50. Anıl, D.; Berksun, E.; Durmuş-Sayar, A.; Sevinis, E. B.; Ünal, S. Recent advances in waterborne polyurethanes and their nanoparticle-containing dispersions. Handbook of Waterborne Coatings 2020, 249302, https://doi.org/10.1016/B978-0-12-814201-1.00011-1.

51. Zhang, Y.; Zhang, W.; Wang, X.; Dong, Q.; Zeng, X.; Quirino, R.L.; Lu, Q.; Wang, Q.; Zhang, C. Waterborne polyurethanes from castor oil-based polyols for next generation of environmentally-friendly hair-styling agents. Prog. Org. Coat. 2020, 142, p.105588, https://doi.org/10.1016/j.porgcoat.2020.105588.

52. Lei, Y.; Liu, Z.; Wu, B.; Jiang, L.; Lei, J. Preparation and properties of cross-linked waterborne polyurethane based on solvent-free route. Polym. Bull. 2020, 77, 3263-3275, https://doi.org/10.1007/s00289-019-02918-5. 